\title{
Bilateral Pigmented Paravenous Chorioretinal Atrophy: A Case Report
}

\author{
Sergio E. Hernández-Da Mota Adolfo Chacón-Lara \\ Clínica David, Unidad Oftalmológica, and Ophthalmology Department, General \\ Hospital 'Dr. Miguel Silva', SSA, Morelia, Mexico
}

\section{Key Words}

Atrophy · Chorioretinal atrophy · Paravenous atrophy

\begin{abstract}
Purpose: To describe a case of bilateral pigmented paravenous chorioretinal atrophy. Methods: Observational case report.

Results: A 50-year-old female patient complained of right eye pain and decreased near visual acuity. She had a best-corrected visual acuity of 20/20 in both eyes. In the posterior segment, there were atrophic changes along retinal vessels, and bony spicule pigmentation was observed in a paravenous distribution. There were some abnormal changes in the electroretinogram and electrooculogram in both eyes.
\end{abstract}

Conclusions: A diagnosis of bilateral pigmented paravenous chorioretinal atrophy was made.

\section{Introduction}

Pigmented paravenous chorioretinal atrophy (PPCA) is a bilaterally symmetric stationary disease of the ocular fundus in which bony spicule pigmentation can be seen in a paravenous distribution. A variable degree of chorioretinal atrophy in the same distribution may also be present extending posteriorly and is confluent with zones of atrophy surrounding the optic disc. Additionally, migration of pigment into the retina, surrounding the vessels, may be observed [1-3].

\section{Case Report}

A 50-year-old female patient complained of right eye pain and decreased near visual acuity. She had a best-corrected visual acuity of 20/20 in both eyes. The anterior segment of both eyes was normal. In 
the posterior segment, there were atrophic changes along retinal vessels, and bony spicule pigmentation was observed in a paravenous distribution (fig. 1, fig. 2).

The visual field of the right eye was markedly constricted, especially in the nasal aspect, while that of the left eye was full on Goldmann perimetry. A rod-dominated (scotopic) electroretinogram of the right eye revealed a subnormal b-wave amplitude $(100 \mu \mathrm{V})$ with a normal implicit time $(40 \mathrm{~m} / \mathrm{s})$. The electroretinogram of the left eye, in a rod-dominated condition, showed a b-wave amplitude of $300 \mu \mathrm{V}$ with an implicit time of $40 \mathrm{~m} / \mathrm{s}$. Electrooculography showed a light-peak to dark-trough ratio of 1.8 for the right eye and 1.9 for the left eye. Fluorescein angiography of the right eye revealed an extensive area of choriocapillaris atrophy with prominently visible choroidal vessels along the major retinal veins adjacent to the disc in an early arterial phase. In the arteriovenous phase, hyperfluorescence was visible at the edge of the atrophic area, and hypofluorescence was seen corresponding to the areas of pigment clumping. Systemic examination was unremarkable and serology for syphilis and skin test for tuberculosis were negative.

The patient's informed consent was obtained for the publication of this case report, which follows the guidelines for experimental investigations required by the Institutional Review Board or Ethics Committee of the institutions with which the author is affiliated.

\section{Discussion}

PPCA is an uncommon disease of unknown etiology. The first case with this disorder was described by Brown in 1937 under the name of retinochoroiditis radiata. In 1962, Franceschetti coined the term 'pigmented paravenous choroiditis'. Of the 90 previously reported cases, 80 have been male patients and have mostly been determined to be sporadic [4]. PPCA is bilaterally symmetric, although unilateral cases have been reported [5].

In PPCA, there may be an autosomal dominant pattern caused by a mutation in the CRB1 gene located on chromosome 1q31-q32.1 or an X-linked inheritance pattern, although most cases are sporadic and may represent an acquired response pattern to an infectious or inflammatory disease. Patients are usually asymptomatic, and retinal findings are incidental like in our case. The retinal lesions are relatively stable over time. The optic discs, maculae, and retinal vessels are typically normal, although some cases have demonstrated attenuation of vessels, optic disc pallor, and retinal pigment epithelial changes in the macula. Electroretinographic responses are only mildly to moderately abnormal, if at all, but the electrooculogram is usually affected, often significantly, like in our case. On fluorescein angiography, extension of hyperfluorescence into the retina can be observed, outlining the areas of retinal pigment epithelial atrophy, and in some cases may demonstrate evidence of choriocapillaris atrophy [4].

\section{Acknowledgements}

The author wishes to thank the staff of Clínica David and Dr. Adolfo Chacon-Lara.

\section{Disclosure Statement}

The author has no conflicts of interest in connection with this case report. No financial support was received for the submission of this case report. 


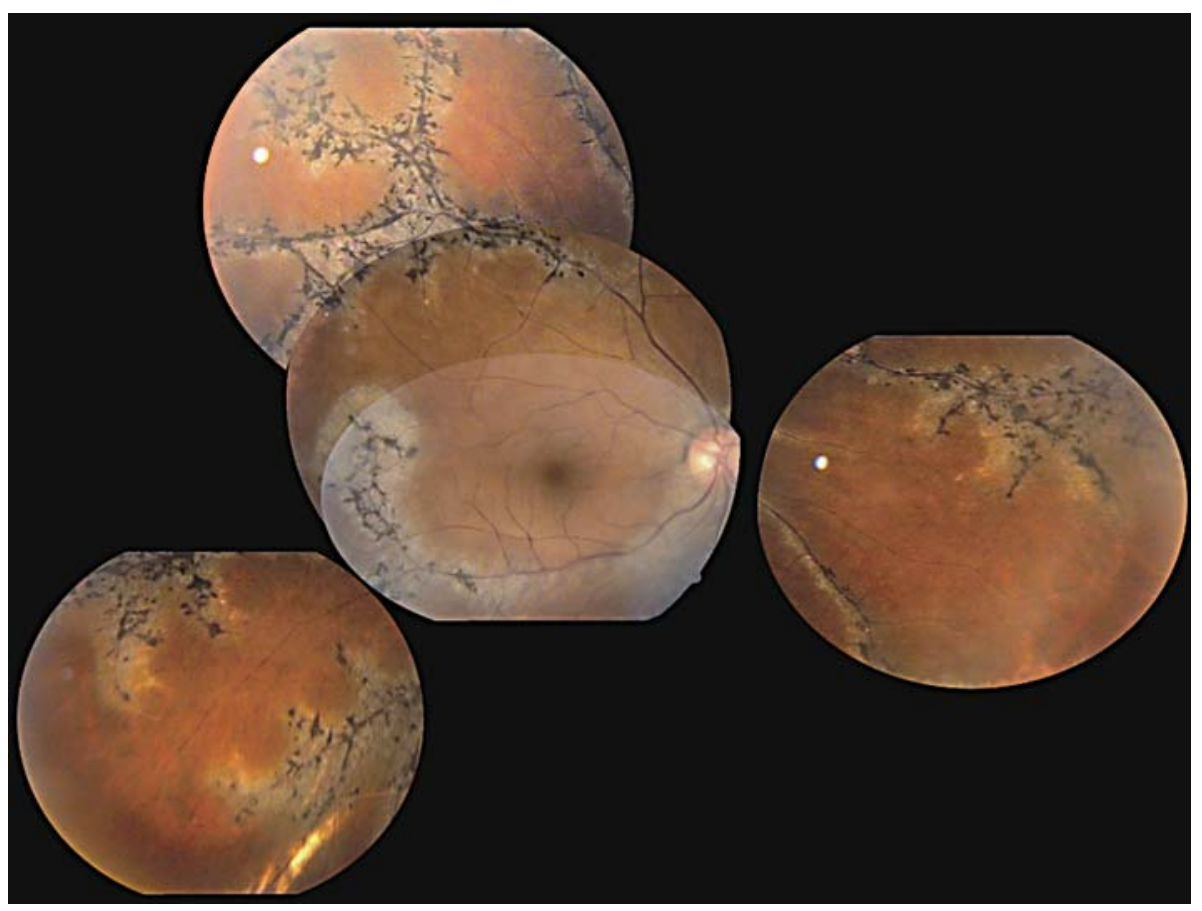

Fig. 1. Clinical composite picture of paravenous atrophy in the right eye with bony spicule pigmentation. 


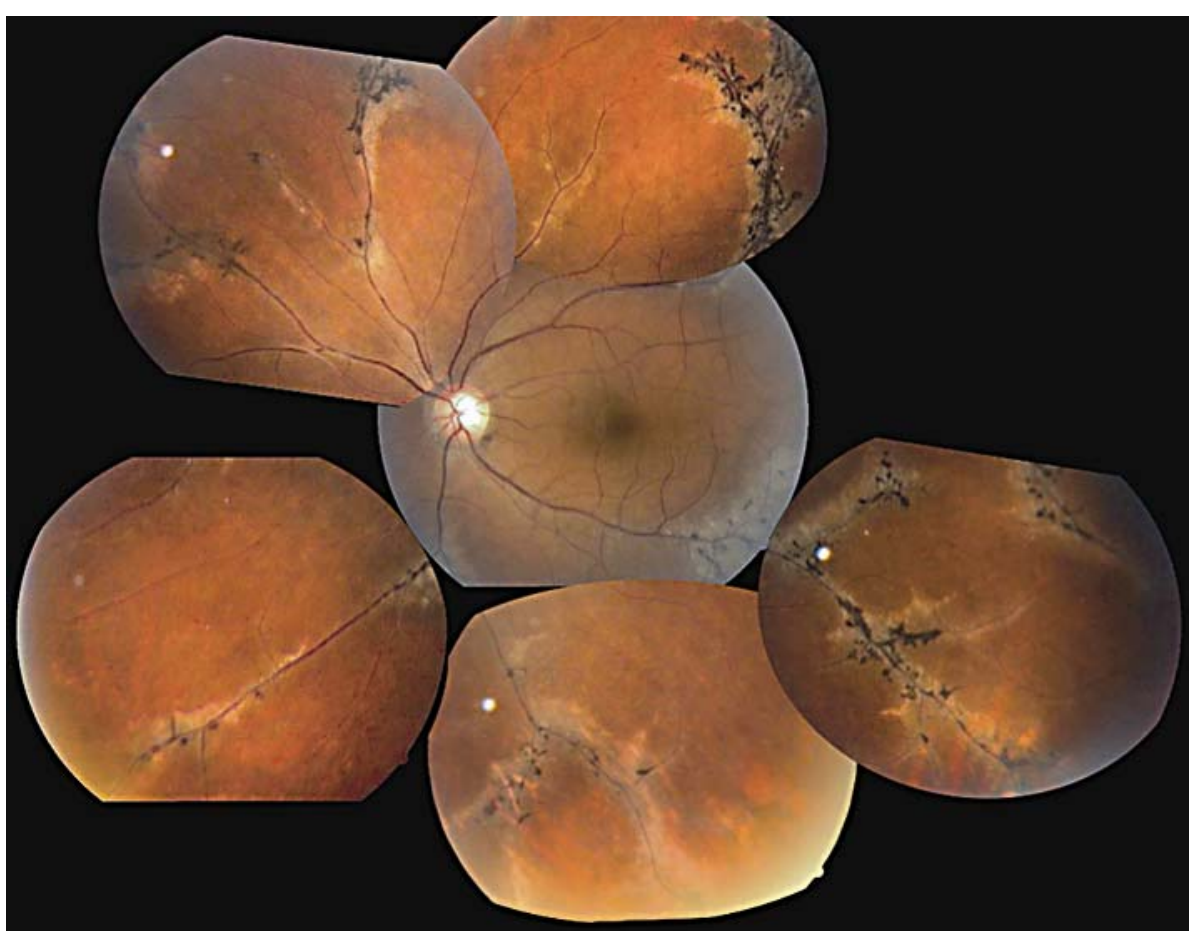

Fig. 2. Left-eye paravenous atrophy.

\section{References}

1 Murray AT, Kirkby GR: Pigmented paravenous retinochoroidal atrophy: a literature review supported by a unique case and insight. Eye 2000;14:711-716.

-2 Bozkurt N, Bavbek T, Kazokoğlu H: Hereditary pigmented paravenous chorioretinal atrophy. Ophthalmic Genet 1998;19:99-104.

-3 Noble KG: Hereditary pigmented paravenous chorioretinal atrophy. Am J Ophthalmol 1989;108:365-369.

-4 McKay GJ, Clarke S, Davis JA, Simpson DA, Silvestri G: Pigmented paravenous chorioretinal atrophy is associated with a mutation within the crumbs homolog 1 (CRB1) gene. Invest Ophthalmol Vis Sci 2005;46:322-328.

-5 Charbel Issa P, Scholl HP, Helb HM, Fleckenstein M, Inhetvin-Hutter C, Holz FG: Unilateral pigmented paravenous retinochoroidal atrophy. Klin Monbl Augenheilkd 2007;224:791-793. 